\title{
GIS and LiDAR Use for Identification of Potential Road Hazard Locations
}

\author{
Anu Pradhan ${ }^{1}$ and William Rasdorf ${ }^{2}$, P.E., F. ASCE
}

\begin{abstract}
In Northerly climates snowstorms are common occurrences that pose severe hazards to drivers. Thousands of lives and property worth of millions of dollars are lost annually. This paper presents a novel method of identifying road segments that pose special hazards to drivers during and after snowstorms by using Light Detection and Ranging (LiDAR) data and a Geographic Information System (GIS).
\end{abstract}

The methodology presented herein can be used for prioritizing roadway snow clearing efforts by identifying the most hazardous road segments. The proposed methodology uses the spatial analysis functionality provided by GIS software and three-dimensional (3D) data obtained from an airborne LiDAR scanning system.

The computing focus of this paper is on spatial data analysis. The data include road centerline 3D coordinates (to identify roadway crests and troughs), aspect data (to determine ground surface slope), and sunlight data (to determine ground surface illumination values and identify shaded areas). Combining and analyzing these data sets identify potentially dangerous north facing, shaded, steep slopes over large geographic areas.

KEYWORDS: GIS, Hazards, Transportation Safety, LiDAR, Response Routing, Traffic Control Devices, Road Hazards, Spatial Analysis, Spatial Data

\footnotetext{
${ }^{1}$ Graduate Student, PH 119, Department of Civil and Environmental Engineering, Carnegie Mellon University, Pittsburgh, PA 15213 Email: pradhan@cmu.edu

${ }^{2}$ Professor, Department of Civil, Construction, and Environmental Engineering, NC State University, Campus Box 7908, Raleigh, NC 27695 Email: rasdorf@ncsu.edu
} 


\section{INTRODUCTION}

Most hazardous road segments during and after snow and ice storms are typically located on roads with steep and north facing slopes. North facing slopes tend to receive less sunlight compared to south facing slopes, causing snow to remain for longer durations and to accumulate in greater volumes. This paper outlines a methodology for identifying these road segments using a Geographic Information System (GIS) and Light Detection and Ranging (LiDAR) point cloud data prior to a severe weather event. The advantage of using this methodology is that it can be applied statewide, it is fully automated, and it requires no field activity or construction efforts. This sets it apart from alternative methods such as the use of field sensors, either embedded in pavement or not.

\section{LiDAR}

LiDAR is an active remote sensing technology that is used to collect topographic data (NOAA 2003). The data are collected with aircraft-mounted lasers capable of recording elevation measurements at a rate of 5,000 to 50,000 pulses per second. A LiDAR dataset is simply a collection of somewhat randomly distributed 3D points. The end product is an accurate, geographically registered longitude, latitude, and elevation from mean sea for every data point [Baltsavias 1999]. Latitude, longitude, and elevation are typically presented in local state plane coordinate systems as north, east, and elevation coordinates.

LiDAR is capable of providing both horizontal and vertical information at high spatial resolutions and accuracies. LiDAR data are accurate to $+/-15 \mathrm{~cm}$ for vertical measurements and +/- $1.5 \mathrm{~m}$ (worst case scenario) for horizontal distances [Flood 1999]. LiDAR technology has been used in many areas of application such as (a) detection of flooded road segments [Cai, Rasdorf, Tilley 2007; Cai, et. al. 2006], (b) determination of 3D road centerline from 2D road centerline [Cai 2003], and (c) detection of hazard posing trees that grow along highways [Laefer and Pradhan 2006].

\section{GIS}

GISs consist of a computer-based tool set for collecting, storing, retrieving, transforming, and displaying spatial data from a discipline-specific domain for a particular set of purposes [Burrough and McDonnell 1998]. GIS is a software system capable of performing complex spatial analysis operations (e.g., proximity analysis and buffering) on geographic data that would otherwise be too repetitive, expensive, and inaccurate to perform by hand [Longley et. al. 2001].

GIS has been used as an enabling technology to solve many transportation problems [Fletcher 1987; Nyerges and Dueker 1988]. It has been used in a wide variety of transportation areas, such as infrastructure planning, design, and management; transportation safety analysis; travel demand analysis; traffic monitoring and control; 
public transit planning and operations; environmental impact assessment; hazard mitigation; and intelligent transportation systems. In the context of this paper, GIS is used to identify hazardous road segments based on their geometry and orientation.

GISs work well with area-based data and land surfaces but they were not originally designed to handle linear data. Transportation systems are roadway networks that are comprised of mostly linear elements. While transportation capabilities have been more recently incorporated into GISs as there is still missing functionality. This paper suggests a solution for one such missing capability.

\section{METHODOLOGY}

Figure 1 illustrates the overall methodology used to perform the required spatial data transformation and analysis. The primary elements of the methodology include:

- Determine the elevations of road segments

- Determine the slope of road segments

- Determine the orientation of road segments with respect to geographic north

- Combine slope, orientation, and location to identify hazardous road segments

\section{Elevation}

Digital road centerline location data is typically available in a planimetric form as maps. Road centerlines are usually represented as a set of $2 \mathrm{D}$ points having $\mathrm{x}$ and $\mathrm{y}$ coordinates (or, more formally, $\mathrm{N}$ and $\mathrm{E}$ coordinates). It is not possible to obtain elevation information from such planimetric data alone. It is possible, however, to utilize National Elevation data and LiDAR to obtain such elevation data. [Cai 2003] demonstrated that LiDAR data points can be used to acquire accurate elevation data to create 3D length of road centerlines. He also compared different existing elevation data sources (such as National Elevation data and Digital Elevation Models) with LiDAR data sources and concluded that LiDAR raw data points are the most accurate among them for transportation applications.

3D elevation surfaces can be generated from LiDAR data points using different techniques such as bilinear interpolation and spline interpolation. Such 3D elevation surfaces are often represented as rectangular or square grids consisting of numerous cells where each cell is assigned an elevation. In one 3D model of an elevation surface, each cell contains an elevation value.

\section{Slope}

Once 3D elevation surfaces are created, one can generate slopes from the given 3D elevation surface. Similar to an elevation surface, a slope surface can also consist of a grid of cells where each cell represents a slope value. The slope is defined as a measure of the rate of change in elevation at a surface location, usually expressed as a percentage. Technical details about calculating slope surface from elevation surface can be found in [Skidmore 1989]. For our purposes slope is determined simply by equation 1. 


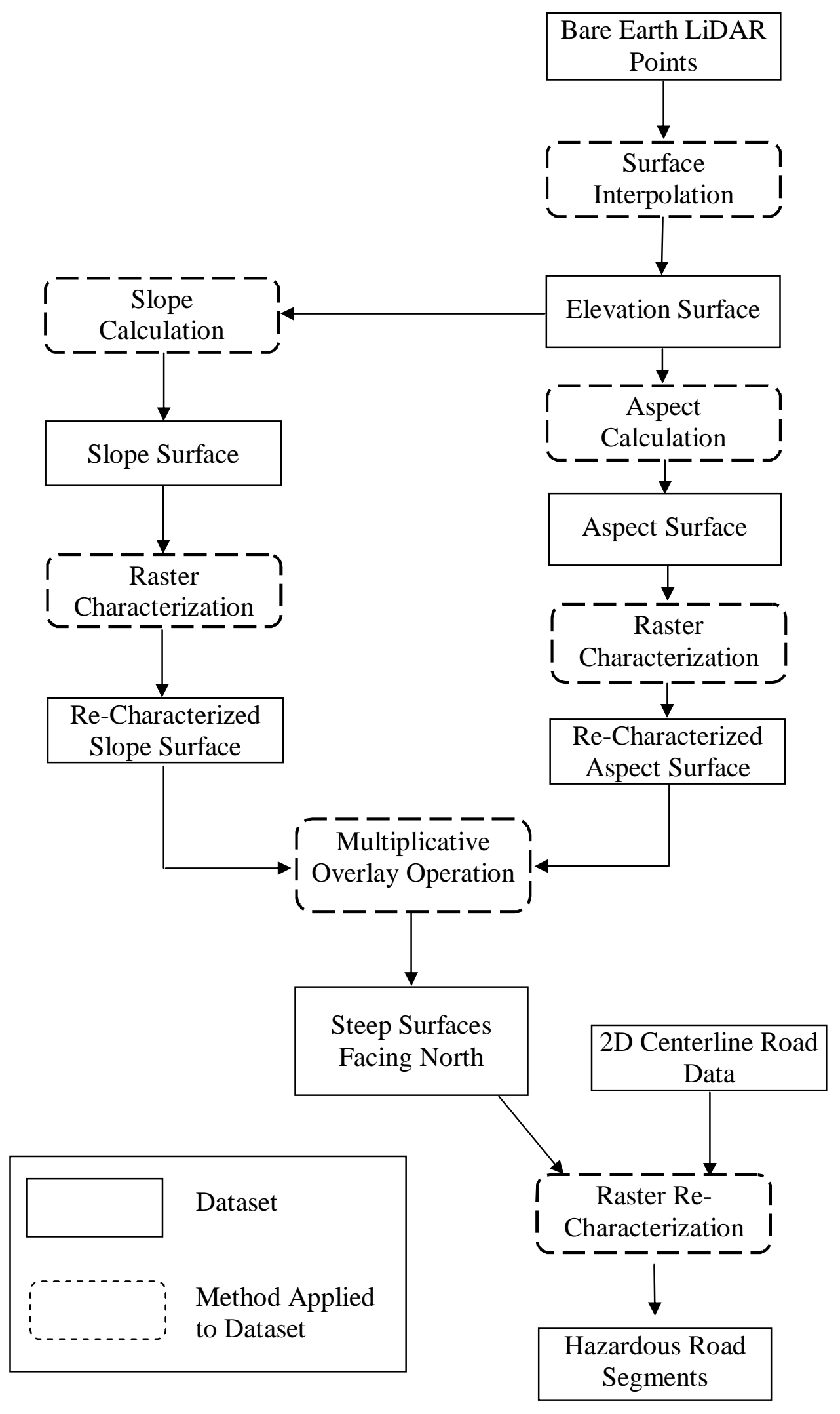

Figure 4. Spatial Analysis Methodology 


$$
\text { Slope }=\text { Rise } / \text { Run } * 100
$$

Once the slopes for different road segments have been determined, one can set a threshold for the slope value (e.g., 10\%), to identify those slopes which are steep.

\section{Orientation}

For our purposes, in addition to determining slope, it is also necessary to identify north facing segments of each road. To identify north facing surfaces, one can compute an aspect surface from the 3D elevation surface. Aspect surfaces are currently being used for different purposes: (a) to identify north facing slopes for ski runs [Pfeffer et. al. 2003] and (b) to identify southerly slopes of mountains and their surrounding residential areas that have a high probability of being hit by water from rapidly melting snow [McCoy and Johnston 2001]. In our study aspect surface was used to find north facing surfaces which are exposed to the least amount of sunlight. Such road segments are vulnerable to snow hazards since it takes a longer time to melt the snow or ice resting on them.

Continuing to use a grid-based model, an aspect surface can be either a rectangular or square grid wherein each cell contains an aspect value identifying the direction that cell faces. The value is measured clockwise in degrees from 0 (due north) to 360 (again due north) completing a full circle. A flat surface, which possesses no aspect direction, is assigned a value of -1 . North facing slopes typically have aspect values ranging from 315 to 45 degrees as shown as shaded region in Figure 2. System users or a given DOT, however, could set other orientations as well depending on their needs Existing GIS software tools support a function that can be used to generate an aspect surface from a given 3D elevation surface.

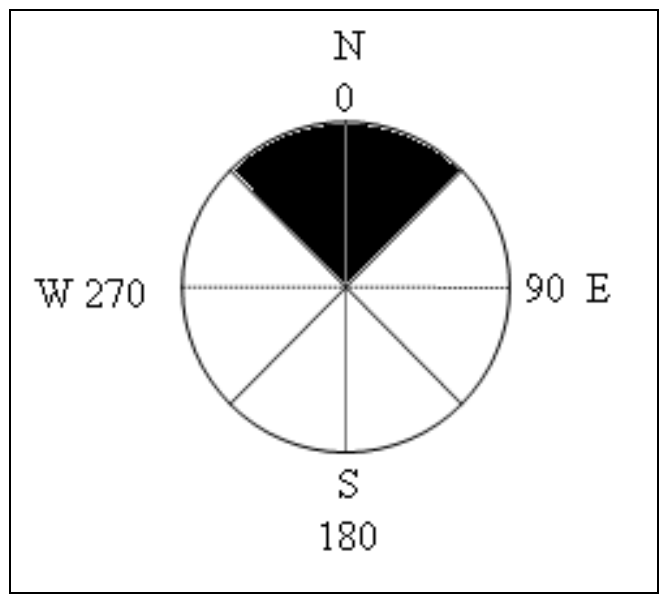

Figure 2. Aspect Values

\section{Hazardous Road Segments Identification}

Hazardous road segments, now defined as having steep north facing slopes, can be identified simply by performing spatial overlays using three data sources (2D road centerline location, slope surface, and aspect surface) and specifying a grid cell size. Prior to performing the spatial overlay, slope surface and aspect surface are re- 
characterized to simplify the spatial overlay process. Re-characterization replaces the current cell value with a new boolean value. For example, if the aspect values for a north facing surface are ascertained to be most critical in a range of aspect value $>$ 315 and $<45$, the aspect surface can be re-characterized into a grid containing 0 and 1 values as shown in Error! Reference source not found.3.

\begin{tabular}{|c|c|c|c|c|}
\hline-1 & 126 & 320 & 26 & 200 \\
\hline-1 & 1 & 319 & 44 & 212 \\
\hline 301 & 201 & 355 & 356 & 183 \\
\hline 1 & 96 & 10 & 10 & 43 \\
\hline 322 & 345 & 112 & 16 & 25 \\
\hline
\end{tabular}$\quad$\begin{tabular}{|c|c|c|c|c|c|}
\hline 0 & 0 & 1 & 1 & 0 \\
\hline 1 & 0 & 1 & 1 & 1 & 0 \\
\hline 1 & 1 & 0 & 1 & 1 \\
\hline
\end{tabular}

Figure 3. Raster Re-Characterization for an Aspect Surface

\begin{tabular}{|l|l|l|l|l|}
\hline 0 & 0 & 1 & 1 & 0 \\
\hline 0 & 1 & 1 & 1 & 0 \\
\hline 0 & 0 & 1 & 1 & 0 \\
\hline 1 & 0 & 1 & 1 & 1 \\
\hline 1 & 1 & 0 & 1 & 1 \\
\hline
\end{tabular}

Aspect Surface

$\div \quad$\begin{tabular}{|c|c|c|c|c|}
\hline 1 & 0 & 1 & 1 & 1 \\
\hline 0 & 1 & 0 & 0 & 1 \\
\hline 0 & 0 & 1 & 1 & 0 \\
\hline 1 & 0 & 1 & 1 & 1 \\
\hline 1 & 1 & 0 & 1 & 1 \\
\hline
\end{tabular}

Slope Surface

\begin{tabular}{|l|l|l|l|l|}
\hline 0 & 0 & 1 & 1 & 0 \\
\hline 0 & 1 & 0 & 0 & 0 \\
\hline 0 & 0 & 1 & 1 & 0 \\
\hline 1 & 0 & 1 & 1 & 1 \\
\hline 1 & 1 & 0 & 1 & 1 \\
\hline
\end{tabular}

Combined Surface

Figure 4. Multiplicative Overlay Operation Output 
Similarly, such re-characterization can be performed for slope surfaces as well. In other words, cells containing values greater than a certain threshold value (say 10\%) are assigned a value of 1 and the remaining cells are assigned a value of 0 . One can use standardized GIS software functions to determine the initial values for both aspect and slope.

Once the re-characterization process is complete, a simple overlay multiplicative operation can be used to combine slope and aspect surface grids to identify steep and north facing surface grids as shown in Figure 4. In Figure 4, the cells containing a value of 1 in the output surface obtained from a multiplicative overlay operation represent north facing and steep roads. The re-characterization process simplified the process of combining slope and aspect surfaces to that of a Boolean operation.

The combined surface model of Figure 4 can then be spatially overlaid with the 2D road centerline to determine the hazardous road segments as shown in Figure 5. The road segments shown as a bold line are hazardous road segments, while the road segments shown as a dashed line are not hazard-posing road segments.

\begin{tabular}{|c|c|c|c|c|}
\hline 0 & 0 & 1 & 1 & 0 \\
\hline 0 & 1 & 0 & 0 & 0 \\
\hline 0 & 0 & 1 & 1 & 0 \\
\hline 1 & 0 & 1 & 1 & 1 \\
\hline 1 & 1 & 0 & 1 & 1 \\
\hline
\end{tabular}

Figure 5. Hazardous and Non-Hazardous Road Segments

While the model presented herein is based simply on a raster model the need at the time the work was done did not justify a more complex model. Using a grid cell model along with overlays was sufficient to make the needed determination. Using this approach in combination with road locations was the unique aspect of the work. At the same time it is clear that this grid-based model can generate a more precise result and significantly enhanced refinement if the grid size is made smaller. It is recommended that a future study be conducted to determine the true impact of the grid size on the result and to determine the appropriate level of accuracy to meet DOT needs. Aspect and slope can certainly aid in the identification of hazardous road segments based on these parameters. 


\section{CONCLUSIONS}

LiDAR data has been shown to have accuracies suitable for transportation applications [Rasdorf et. al. 2004]. LiDAR is useful, in combination with GIS, to determine accurate 3D surface representations and characteristics, particularly for transportation applications.

This paper has shown that it is possible to use surface characteristics to find roadway slopes and orientation. Thus, one is enable to rapidly identify potentially hazardous roadway segments having a high likelihood of being dangerous as a result of snow and ice. Such road segments are those which are steep and which face north. The reader should note that this is an activity that occurs prior to the hazardous event. That is, it is an activity that can be done on a one time basis, after which all the hazardous segments will have been determined.

Once hazardous road segments are identified, it is possible to initiate responses that mitigate the dangers to drivers. A reactive response might be to direct maintenance personnel to these road segments to quickly initiate remediation methods, such as the application of salt, chloride, or ash to the road surface. A preemptive response could be to ensure that an appropriate set of traffic control devices, such as warning signs, are in place to draw attention to the potential hazards. Finally, a long term response might also be to modify the roadway geometry to reduce its slope and/or change its orientation.

This work has demonstrated that, using existing data and software, a unique transportation problem can be effectively addressed. However, additional work needs to be done. The authors recommend that studies be conducted to utilize different ranges of aspect values and different thresholds of slope to assess the sensitivity of the results to these variables. Furthermore, it is also recommended that more work be done to further utilize road horizontal geometry in this scenario. It is clear that road locations that are both steep and sharp are the most critical. A factor needs to be defined and determined that simultaneously takes into account both slope and curvature. Using GIS this should be an automated procedure. Additionally, in a broader context, it should be possible to use the basic ideas presented herein to identify potentially hazardous curves and to assess drivers' lines of sight thereby contributing to other transportation safety needs.

Finally, the methodology presented herein used a raster (grid-based) model wherein slope, aspect, and location values were converted to Boolean values wherein a 1 meant steep, north facing, on the road and a 0 meant not steep, not north facing, and not on the road. The basic primitive was a grid cell. Alternatively, the authors recommend another approach using a standard size road segment as a basis. (Its length could then be altered to perform a sensitivity analysis just as the size of the raster grid can be altered.) For that segment itself a slope and aspect can be generated. The same limits could be used for this proposed approach as for the original. 


\section{ACKNOWLEDGEMENTS}

The authors would like to acknowledge the contributions of members of the North Carolina Department of Transportation GIS Unit for their guidance and for the use of NCDOT data and software. These individuals include Chris Tilley, William Miller, and Forrest Robson.

\section{REFERENCES}

Baltsavias, E. P. (1999). "Airborne Laser Scanning: Basic Relations and Formulas." Journal of Photogrammetry and Remote Sensing, International Society of Photogrammetry and Remote Sensing, Volume 54, Pages 199-214.

Burrough, P. A. and McDonnell, R. A. (1998). Principles of Geographic Information Systems-Errors and Quality Control. Oxford University Press, New York, Pages 220-240.

Cai, H. (2003). "Accuracy Evaluation of a 3-D Spatial Modeling Approach to Model Linear Objects and Predict Their Lengths." PhD Dissertation, North Carolina State University, Raleigh, NC, December.

Cai, H., Rasdorf, W., and Tilley, C. (2007). "An Approach to Determine the Extent and Depth of Highway Flooding." Journal of Infrastructure Systems, American Society of Civil Engineers, Volume 13, Number 2, Pages 157-167.

Cai, H., Rasdorf, W., Tilley, C., Smith, L. C., and Robson, F. (2006). "Geographic Information Systems/National Elevation Data Route Mileage Verification." Journal of Surveying Engineering, American Society of Civil Engineers, Volume 132, Number 1, Pages 40-49.

Fletcher, D. R. (1987). "Modeling GIS Transportation Networks." Proceedings of the 25th Annual Conference, Urban and Regional Information Systems Association, Fort Lauderdale, FL, Pages 84-92.

Flood, M. (1999). "Commercial Development of Airborne Laser Altimetry." Proceedings of the Workshop on Mapping Surface Structure and Topography by Airborne and Space Borne Lasers, International Society of Photogrammetry and Remote Sensing, Sydney, Australia, Pages 13-20.

Laefer, D. F. and Pradhan, A. R. (2006). "Evacuation Route Selection Based on TreeBased Hazards Using LiDAR and GIS." Journal of Transportation Engineering, American Society of Civil Engineers, Volume 132, Number 4, Pages 312-320.

Longley, P. A., Goodchild, M. F., Maguire, D. J., and Rhind, D. W. (2001). Geographic Information Systems and Science, Wiley, Chichester, U.K. 
Natural Oceanic and Atmospheric Administration (NOAA). (2008). "About LiDAR Data." <http://www.csc.noaa.gov/products/sccoasts/html/tutlid.htm> (Nov. 25, 2008).

McCoy, J. and Johnston, K. (2001). Using ArcGIS Spatial Analyst, ESRI Press, Redmond, CA

Nyerges, T. L. and Dueker, K. J. (1988). "Geographic Information Systems in Transportation." Report HPN-22, Office of Planning, Federal Highway Administration, Washington, D.C.

Pfeffer, K., Pebesma, E., and Burrough, P. (2003). "Mapping Alpine Vegetation Using Vegetation Observations and Topographic Attributes." Landscape Ecology, Volume 18, Number 8, Pages 759-776.

North Carolina Flood Mapping Program (NCFMP). (2008). "North Carolina Flood Mapping Program." <http://www.ncfloodmaps.com> (Nov. 30, 2008).

Rasdorf, W., Cai, H., Tilley, C., Brun, S., and Robson, F. (2004). "Accuracy Assessment of Interstate Highway Length Using Digital Elevation Models." Journal of Surveying Engineering, American Society of Civil Engineers, Volume 130, Number 3, Pages 142-150.

Skidmore, A. K. (1989). "A Comparison of Techniques for Calculating Gradient and Aspect From a Gridded Digital Elevation Model." Taylor \& Francis, Pages 323 - 334. 